\title{
Measurement of charmonium photoproduction in ultra-peripheral collisions with the ALICE detector at the LHC
}

Andrea Agostinelli*†

University of Bologna and INFN Bologna, Italy

E-mail: andrea.agostinelli@bo.infn.it

Ultra-relativistic heavy ions generate strong electromagnetic fields which offer the possibility to study gamma-gamma and gamma-nucleus processes at the LHC in so called ultra-peripheral collisions (UPC). The photoproduction of $J / \psi$ vector mesons in UPC is sensitive to the gluon distribution of the interacting nuclei. Here we report on ALICE results of $J / \psi$ coherent and incoherent production measured in $\mathrm{Pb}-\mathrm{Pb}$ collisions at $\sqrt{s_{N N}}=2.76 \mathrm{TeV}$. The $J / \psi$ has been measured via its leptonic decay both in the rapidity range $-3.6<y<-2.6$ and $-0.9<y<0.9$. The results at forward rapidity have been recently published in [1], while for the central region we show a preliminary study.

International Winter Meeting on Nuclear Physics,

21-25 January 2013

Bormio, Italy

* Speaker.

${ }^{\dagger}$ For the ALICE Collaboration 


\section{Ultra-peripheral collisions}

Ultra-peripheral collisions may occur when two accelerated nuclei are separated by impact parameters larger than the sum of their radii: hadronic interactions are strongly suppressed so that both pure electromagnetic and photonuclear processes can be studied. These interactions can be studied in $\mathrm{Pb}-\mathrm{Pb}$ collisions at the LHC at unprecedentedly high energies, where the available center of mass energy per nucleon pair is $2.76 \mathrm{TeV}$, allowing $\gamma \gamma$ interactions at $\sqrt{s_{\gamma \gamma}^{\max }} \sim 80 \mathrm{GeV}$ and $\gamma p$ at $\sqrt{s_{\gamma p}^{\max }} \sim 480 \mathrm{GeV}$, to be compared with 10 and $30 \mathrm{GeV}$ reached at RHIC, respectively. An accelerated charge can be considered as a source of quasi-real photons, according to the proposal by Fermi [2] and the relativistic version of Weizsäcker [3] and Williams [4]. One of these quasi-real photons may collide either with the opposite ion or with one of its quasi-real photons. The cross section for photon induced reactions is large because the strong electromagnetic field of the nucleus enhances the intensity of the virtual photon flux, which grows as $Z^{2}$, where $\mathrm{Z}$ is the charge of the nucleus. The virtuality of the photons is restricted by the nuclear form factor to be of the order $1 / R \sim 30 \mathrm{MeV} / c$ ( $R$ is the radius of the nucleus). UPCs are characterized by the photoproduction of vector mesons $\left(\rho^{0}, J / \psi, \psi^{\prime}, \Upsilon, \ldots\right)$ and pure QED processes giving rise to a di-lepton continuum. The physics of ultra-peripheral collisions is reviewed in $[5,6]$.

\section{Physics motivations}

Exclusive photoproduction of vector mesons, where a vector meson but no other particles are produced in the event, is of particular interest. Exclusive production of $J / \psi$ in photon-proton interactions, $\gamma+p \rightarrow J / \psi+p$, has been successfully modeled in perturbative QCD in terms of the exchange of two gluons with no net-colour transfer [7]. Experimental data on this process from HERA have been used to constrain the proton gluon-distribution at low Bjorken- $x$ [8]. Exclusive vector meson production in heavy-ion interactions is expected to probe the nuclear gluondistribution [9], for which there is considerable uncertainty in the low- $x$ region [10]. A $J / \psi$ produced at rapidity $y$ is sensitive to the gluon distribution at $x=\left(M_{J / \psi} / \sqrt{s_{N N}}\right) e^{ \pm y}$. As an example, at forward rapidities considered here, $-3.6<y<-2.6$, the relevant values of $x$ are $\approx 10^{-2}$ and $\approx 10^{-5}$. Exclusive photoproduction can be either coherent, where the photon couples coherently to all nucleons, or incoherent, where the photon couples to a single nucleon. Coherent production is characterized by low vector meson transverse momentum $\left(\left\langle p_{T}\right\rangle \simeq 60 \mathrm{MeV} / c\right)$ and the target nucleus normally does not break up. Incoherent production, corresponding to quasi-elastic scattering off a single nucleon, is characterized by a higher transverse momentum $\left(\left\langle p_{T}\right\rangle \simeq 500 \mathrm{MeV} / c\right)$ and the target nucleus normally breaks up, but except for single nucleons or nuclear fragments in the very forward region no other particles are produced.

In what follows, results on coherent $J / \psi$ in the forward region detected via its muon decay and coherent $J / \psi$ at central rapidities in both leptonic decays are presented.

\section{Analysis of the collected sample with the ALICE detector}

\subsection{ALICE detector for UP studies}

The ALICE detector [11] allows a study of UPCs in different rapidity ranges, probing different 
Bjorken- $x$ scales. It consists of a central barrel placed in a large solenoid magnet $(B=0.5 \mathrm{~T})$, covering the pseudorapidity region $|\eta|<0.9$, and a muon spectrometer at forward rapidity, covering the range $-4.0<\eta<-2.5$.

The muon spectrometer measures muons produced with a pseudorapidity, $\eta$, within $-4.0<\eta<$ -2.5 . It consist of an absorber of ten interaction lengths, followed by five tracking stations made of two planes of cathode pad MWPC, where the third station is inside a dipole magnet with $3 \mathrm{Tm}$ integrated magnetic field. The spectrometer has a triggering system to select muon candidates with a transverse momentum larger than a programmable threshold.

For the central rapidity analysis the following detectors of the central barrel have been used. The Silicon Pixel Detector (SPD) makes up the two innermost layers of the ALICE Inner Tracking System (ITS), covering extended pseudorapidity ranges $|\eta|<2$ and $|\eta|<1.4$ for the inner and outer layers, respectively. It is a fine granularity detector, having about 107 elements, and can be used for triggering purposes. The Time Projection Chamber (TPC), instrumented with 560000 readout pads from Multi-Wire-Proportional-Chambers (MWPCs), is used for high precision tracking and for particle identification. The TPC acceptance covers the pseudorapidity region $|\eta|<0.9$. Beyond the TPC, the Time-of-Flight detector (TOF) is a large cylindrical barrel of Multigap Resistive Plate Chambers (MRPCs) with about 150000 readout channels. It is used for event triggering and particle identification thanks to its high precision timing measurements for tracks traversing it. Its pseudorapidity coverage matches that of the TPC. Still further out from the interaction region, the Electromagnetic Calorimeter (EMCAL) is a Pb-scintillator sampling calorimeter at a distance of $\sim 4.5$ meters from the beam line, covering the range $|\eta| \leq 0.7$ and a difference in azimuth $\Delta \Phi=100^{\circ}$. It provides 20.1 radiation lengths and consists of 11520 towers.

Both analysis also make use of forward detectors. The VZERO counters consist of two arrays of 32 scintillator tiles each, covering the range $2.8<\eta<5.1$ (VZERO-A, on the opposite side of the muon arm) and $-3.7<\eta<-1.7$ (VZERO-C, on the same side as the muon arm) and positioned respectively at $z=340 \mathrm{~cm}$ and $z=-90 \mathrm{~cm}$ from the interaction point. The Forward Multiplicity Detector (FMD) consists of Si-strip sensors with a total of 51240 active detection elements, arranged in five rings perpendicular to the beam direction, covering the pseudorapidity ranges $-3.4<\eta<-1.7$ (FMD- 3) and $1.7<\eta<5.1$ (FMD-1 and FMD-2), a similar coverage to that of the VZERO detector. Finally, two sets of hadronic zero degree calorimeters (ZDC) are located at $116 \mathrm{~m}$ on either side of the interaction point. The ZDCs detect neutrons emitted in the very forward region, such as those produced by electromagnetic dissociation.

\subsection{Triggers and data analysis}

The measurements were performed during 2011 ALICE data taking with $\mathrm{Pb}-\mathrm{Pb}$ collisions at $\sqrt{s_{N N}}=2.76 \mathrm{TeV}$. Events from two-photon or photonuclear vector meson production were collected by two dedicated triggers implemented tagging events with only two tracks in an otherwise empty detector ( Fig. 1). The following event characteristic is required to trigger events at forward rapidity: (i) a single muon trigger above a $1 \mathrm{GeV} / c p_{T}$-threshold; (ii) at least one hit in the VZERO$\mathrm{C}$ detector since the muon spectrometer covers a similiar pseudorapidity acceptance; (iii) no hits in the VZERO-A detector to reject hadronic collisions. This trigger allows to cover an acceptance in pseudorapidity equal to $-4<\eta<-2.5$, getting a final observed rapidity of $-3.6<y<-2.6$. As far as the central barrel trigger is concerned, a topological request in the angular distribution 

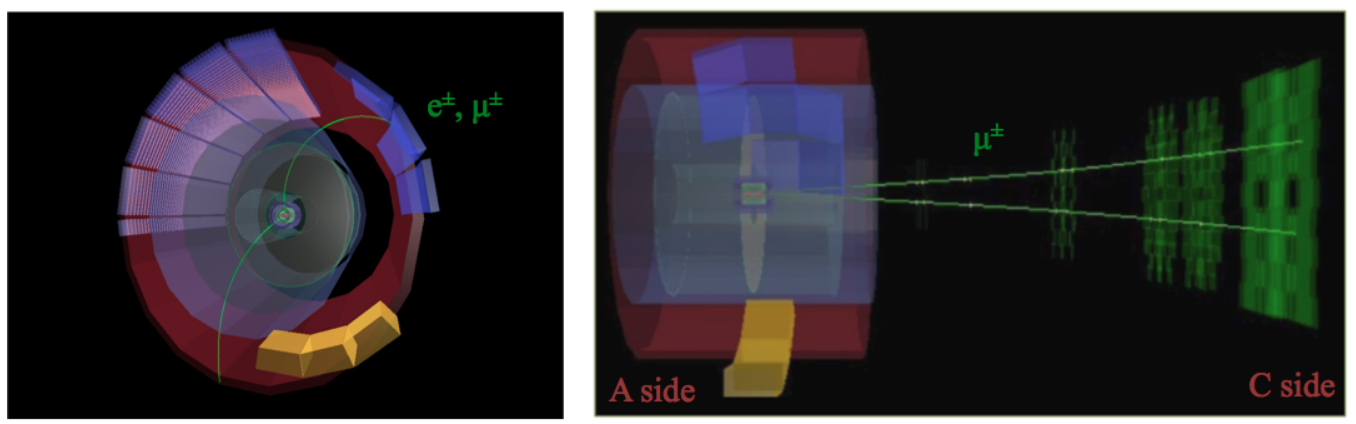

Figure 1: Two examples of triggered events by the central barrel triggering system (left) and the muon spectrometer (right).

of the two tracks has been used since the vector mesons are produced in almost a back-to-back configuration. The complete set of requirements is: (i) at least two hits in the SPD detector; (ii) a number of fired trigger pad in the TOF detector in the range $2 \leq N_{T r g}^{O N} \leq 6$, with at least two of them with a difference in azimuth, $\Delta \Phi$, in the range $150^{\circ} \leq \Delta \Phi \leq 180^{\circ}$; (iii) no hits in the VZERO-A and no hits in the VZERO-C detectors. The acceptance in rapidity corresponds to $|y|<0.9$.

The collected samples are filtered with several offline cuts, described in detail in [1] for the analysis in the forward region. For both analysis, the relevant cuts ensure the rejection of beam-gas and low multiplicity hadronic interactions by using respectively the VZERO timing capabilities and the ZDC count of the emitted neutrons (less than 6 neutrons for coherent events). Moreover, the muon/electron identification via TPC energy loss is mandatory for the central barrel analysis. Non hadronic events with two opposite sign leptons coming from a primary vertex are good candidates for two-photon processes $\left(\gamma \gamma \rightarrow e^{+} e^{-} / \mu^{+} \mu^{-}\right)$or $J / \psi$ photoproduction. In particular, photon processes give a di-lepton exponential background overlapping the $J / \psi$ peak. Finally, different coherent cuts on the di-lepton transverse momentum are adopted: for the forward $J / \psi \mathrm{s}$, coherence condition is fulfilled with transverse momenta below $300 \mathrm{MeV} / c$ while, for the central $J / \psi$ analysis, a coherent cut of $p_{T}<200$ or $p_{T}<300 \mathrm{MeV} / c$ is applied according to the muon or electron decay channels, respectively.

\section{Results and comparison with models}

\subsection{Forward rapidity region}

The results for the forward rapidity region have been recently published in [1]. The mass distribution for the selected events and the transverse momentum distribution for events in the mass range $(2.8,3.4) \mathrm{GeV} / c^{2}$ are shown in Fig. 2. In the invariant mass distribution plot, a peak at the $J / \psi$ mass is clearly seen and has been fitted with an exponential plus a Crystal Ball parameterization. The parameters of the minimum likelihood fit are consistent with expectations from the production of $J / \psi$ vector mesons and the di-muon continuum. The transverse momentum distribution of the $J / \psi$ candidates are well reproduced by Monte Carlo templates which take into account the contributions from coherent and incoherent $J / \psi$ production, the feed-down from $\psi^{\prime}$ and the contribution from the di-muon continuum. As expected, at low transverse momentum the coherent production dominates. The yield of coherent $\mathrm{J} / \psi$ s, obtained by a cut on di-muon momentum below 

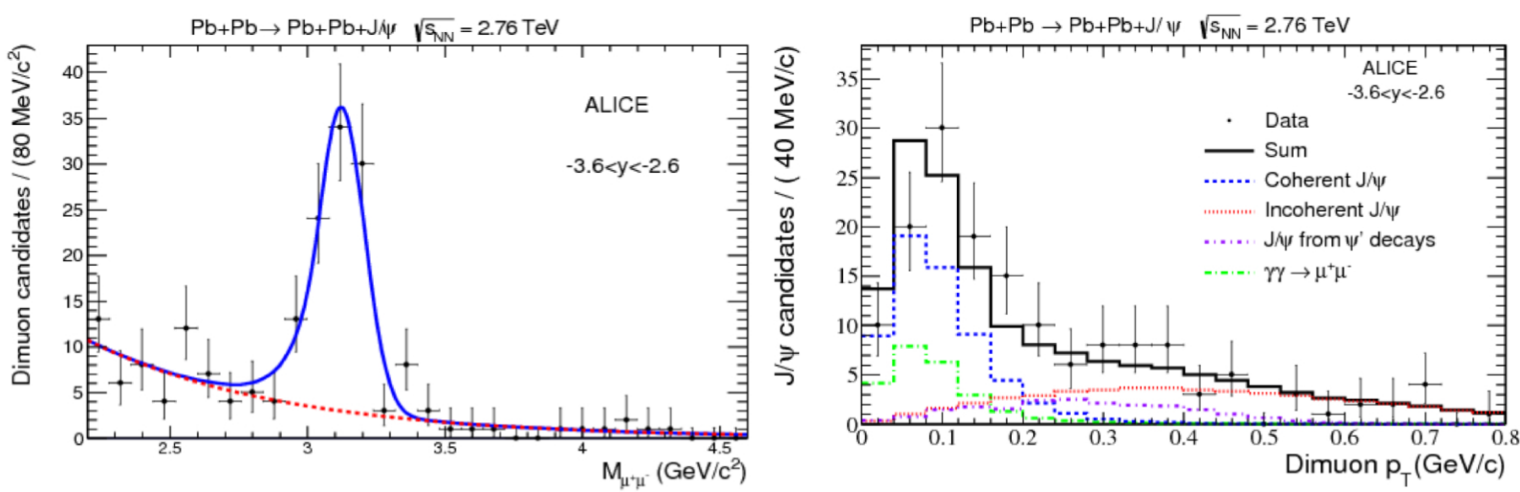

Figure 2: Invariant mass spectrum of forward coherent $J / \psi \rightarrow \mu^{+} \mu^{-}$candidates with $p_{T}<300 \mathrm{MeV} / c$ and less than 6 neutrons in the ZDC (top) and $J / \psi p_{T}$ distribution (bottom) fitted with a MC template as described in the text.

$300 \mathrm{MeV} / c$ and a ZDC cut tuned to select events with less than 6 emitted neutrons, is estimated to be

$$
N_{J / \psi}^{c o h}=78 \pm 10(\text { stat })_{-11}^{+7}(\text { syst }) .
$$

The coherent differential cross section at $\sqrt{s_{N N}}=2.76 \mathrm{TeV}$ in the rapidity interval $-3.6<y<-2.6$ can be written as

$$
\frac{d \sigma_{J / \psi}^{c o h}}{d y}=\frac{1}{B . R .\left(J / \psi \rightarrow \mu^{+} \mu^{-}\right)} \frac{N_{J / \psi}^{c o h}}{N_{\gamma \gamma}} \frac{(A c c \times \varepsilon)_{\gamma \gamma}}{(A c c \times \varepsilon)_{J / \psi}} \frac{\sigma_{\gamma \gamma}}{\Delta y}
$$

where B.R. is the branching ratio in the muon channel, $(A c c \times \varepsilon)$ is the correction for efficiency and acceptance of the coherent and continuum samples, $\Delta y$ is the width of the rapidity interval and $N_{\gamma \gamma}$ is the number of events in the two mass regions neighbouring the $J / \psi$ peak. $\sigma_{\gamma \gamma}$ is the cross section for the continuum production, derived from pure QED calculation. This cross section is known with a precision of about $20 \%$ representing the largest source of systematic errors. In fact the QED coupling gets a factor $Z$ (the charge of the ion) and there are uncertainties on the minimum momentum transfer and the nuclear form factor. The obtained cross section is

$$
\frac{d \sigma_{J / \psi}^{c o h}}{d y}=1.00 \pm 0.18(s t a t)_{-0.26}^{+0.24}(s y s t) m b
$$

\subsection{Mid-rapidity region}

For the central rapidity region, preliminary results are reported. The invariant mass spectrum is shown in Fig. 3, separated in the coherent (top plots), incoherent samples (bottom plots) and decay channels, muons (right), electron (left) . Data are fitted to an exponential curve and a Crystal Ball function. In the muon channel the coherence condition applied requires momenta below 200 $\mathrm{MeV} / c$. The enriched coherent sample, besides the well-defined $J / \psi$ peak, shows also a $\psi^{\prime}$ peak arising from the di-muon continuum. The continuum for the incoherent enriched sample for the muon channel (bottom, right) is likely to come from misidentified $\pi^{+} \pi^{-}$pairs. In the electron channel the effects of the bremsstrahlung due to the detector material is visible in the width and 

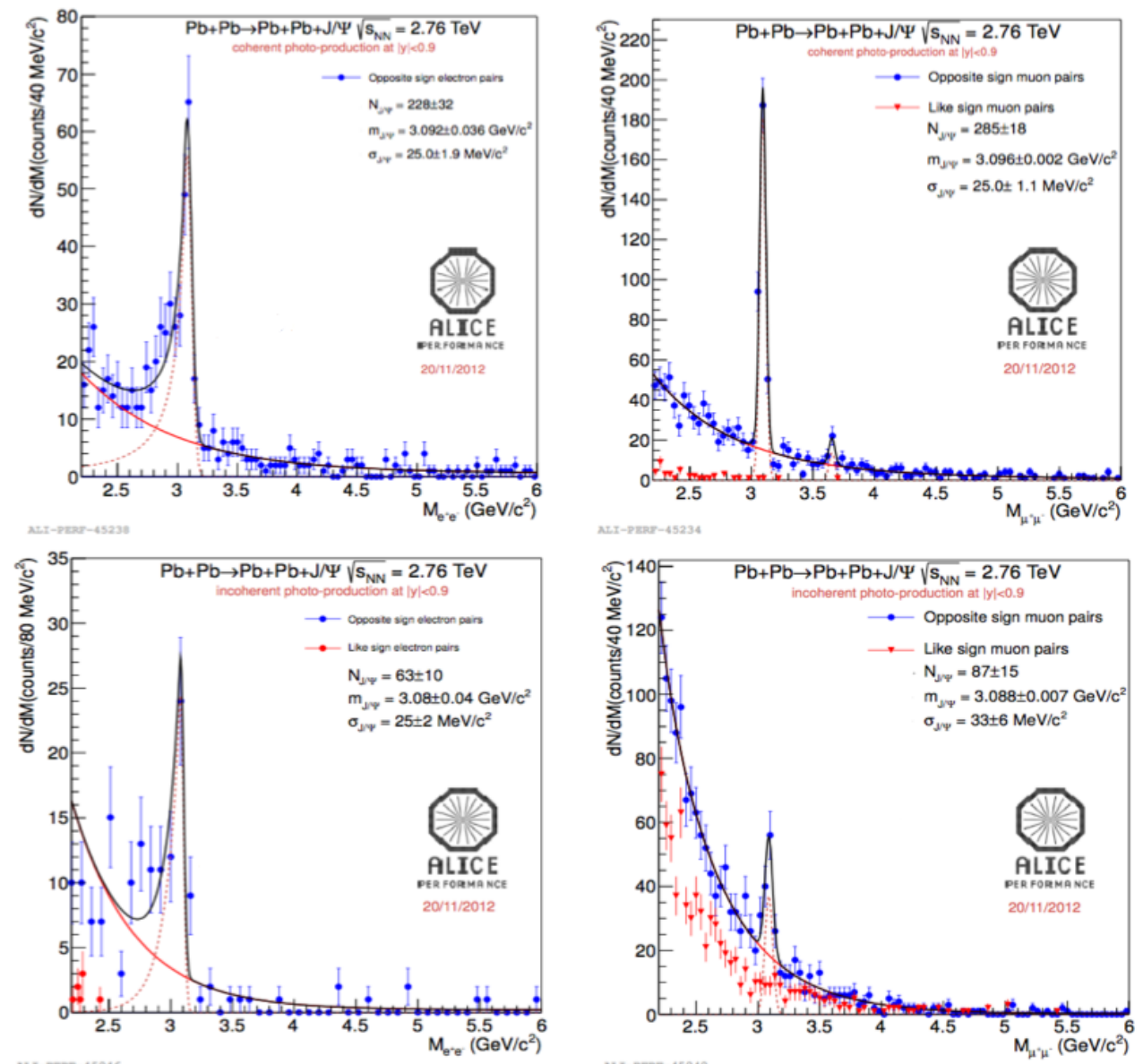

Figure 3: Invariant mass distributions for ultra-peripheral $\mathrm{Pb}-\mathrm{Pb}$ collisions at $\sqrt{s_{N N}}=2.76 \mathrm{TeV}$ and $|y|<0.9$ for events satisfying the event selection, in the invariant mass range $2.2<M_{i n v}<6 \mathrm{GeV} / c^{2}$. Coherent enriched sample (top) and incoherent enriched sample (bottom) for electrons (left) and muons (right). The coherent request is fulfilled with transverse momenta below $200 \mathrm{MeV} / c$ (muon channel) or $300 \mathrm{MeV} / c$ (electron channel) and less than 6 neutrons in the ZDC. Blue (red) circles (triangles) are opposite-sign (likesign) pairs. The continuous line is the result of the fit as described in the text.

asymmetry of the signal and the coherence conditions is better fulfilled with a cut at $300 \mathrm{MeV} / c$. In both cases always less than 6 neutrons in the ZDC are required to get the coherence condition. In Fig. 4 the transverse momentum distribution of di-electrons and di-muons is reported. The Monte Carlo templates reproduce well the data when one takes into account the contributions from coherent and incoherent $J / \psi$ production, the feed-down from coherent and incoherent $\psi^{\prime}$, the contribution of di-lepton continuum and finally a contribution from hadronic $J / \psi$ coming out from very peripheral events.

\subsection{Discussion}

The results obtained at forward and central rapidities can be compared to Monte Carlo predictions, shown in Fig. 5. Several theoretical predictions are presented in this plot. STARLIGHT [12], uses a GVDM coupled to a Glauber approach to link the $\gamma p$ to the $\gamma A$ cross section, the first 

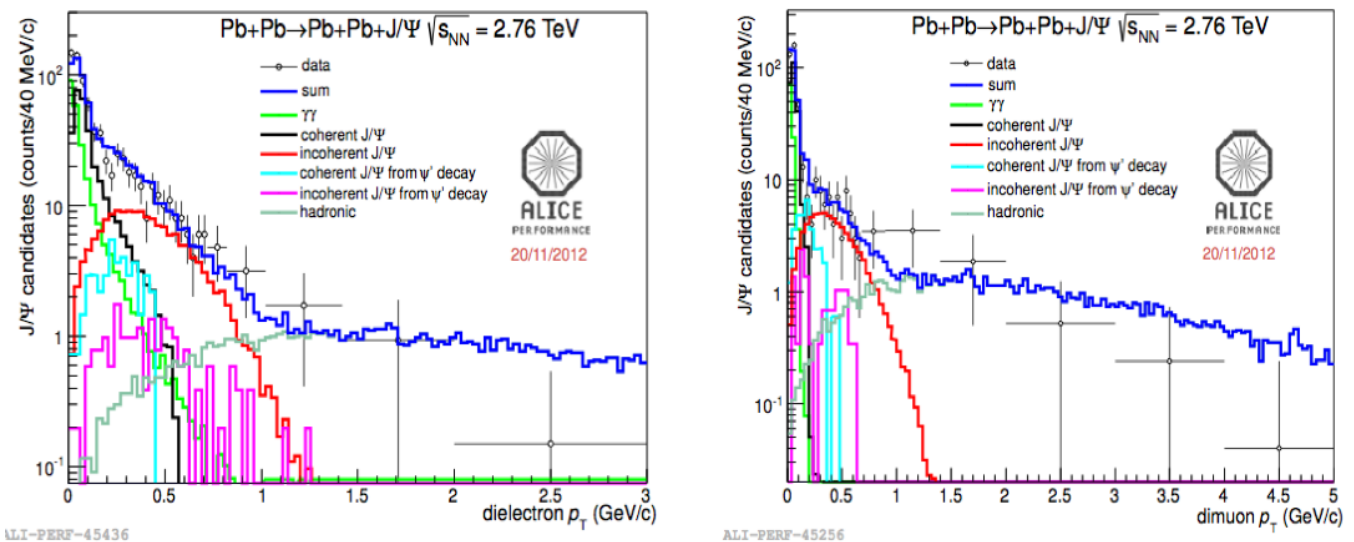

Figure 4: Transverse momentum distribution for the selected $J / \psi$ candidates in the electron (left) and muon (right) channels fitted with Monte Carlo templates as described in the text.

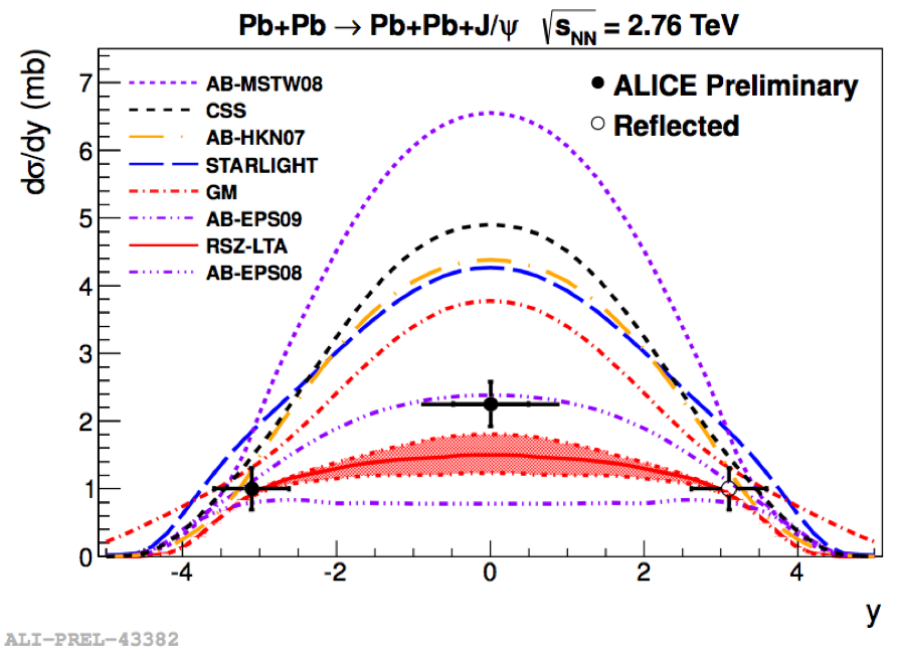

Figure 5: Comparison among the published value of the cross section at forward rapidity [1], the preliminary result at central rapidities and several theoretical models. The error is the quadratic sum of the statistical and systematic errors. More details in the text.

obtained from a parameterization of HERA data. GM [13] is based on the color dipole model, where the scattering amplitude depends on the nuclear profile and the dipole nucleon cross section. The CSS model [14] uses a Glauber approach and the color dipole nucleon amplitude based on the unintegrated gluon distribution of the proton. The AB models [15] use the LO pQCD amplitude scaled to fit $\gamma+p \rightarrow J / \psi+p$ data. For the gluon distribution, AB-MSTW08 assumes no nuclear effects. The other AB models incorporate nuclear effects according to the EPS08, EPS09 or HKN07 prescriptions. Finally, RSZ-LTA [16] is based on the LO pQCD amplitude for two gluon exchange where the nuclear gluon density incorporates shadowing computed in the leading twist approximation. The preliminary result in the central rapidity region is compatible with that in the forward region; the models with no nuclear gluon shadowing are disfavoured, while the AB-EPS09 gives the best prediction. 


\section{Acknowledgments}

I would like to thank the organizers of the 2013 International Winter Meeting on Nuclear Physics for the opportunity to present this work in such a wonderful place as Bormio.

\section{References}

[1] B. Abelev et al. [ALICE Collaboration], Phys. Lett. B 718 1273-1283 (2013).

[2] E. Fermi, Nuovo Cimento 2 143-158 (1925).

[3] C. F. Weizsäcker, Z. Phys. 88 612-625 (1934).

[4] E. J. Williams, Phys. Rev. 45 729-730 (1934).

[5] A. J. Baltz et al., Phys. Rept. 4581 (2008).

[6] C. A. Bertulani, S. R. Klein, J. Nystrand, Ann. Rev. Nucl. Part. Sci. 55271 (2005).

[7] L. Frankfurt, W. Koepf, M. Strikman, Phys. Rev. D 57512 (1998).

[8] A. D. Martin, C. Nockles, M.G. Ryskin, T. Teubner, Phys. Lett. B 662252 (2008).

[9] V. Rebyakova, M. Strikman, M. Zhalov, Phys. Lett. B 710647 (2012).

[10] K. J. Eskola, H. Paukkunen, C. A. Salgado, JHEP 0904065 (2009).

[11] K. Aamodt et al. [ALICE Collaboration], JINST 3 S08002 (2008).

[12] S. R. Klein and J. Nystrand, Phys. Rev. C 60014903 (1999).

[13] V. P. Goncalves and M. V. T. Machado, Phys. Rev. C 84011902 (2011).

[14] A. Cisek, W. Schafer and A. Szczurek, Phys. Rev. C 86014905 (2012).

[15] A. Adeluyi and C. A. Bertulani, Phys. Rev. C 85044904 (2012).

[16] V. Rebyakova, M. Strikman and M. Zhalov, Phys. Lett. B 710647 (2012). 